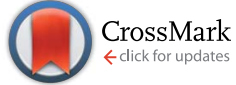

Cite this: J. Mater. Chem. A, 2016, 4, 15638

Received 16th June 2016

Accepted 14th September 2016

DOI: $10.1039 / \mathrm{cta0} 05055 \mathrm{~g}$

www.rsc.org/MaterialsA

\section{Layered 2D alkyldiammonium lead iodide perovskites: synthesis, characterization, and use in solar cells $\dagger$}

\author{
Majid Safdari, ${ }^{a}$ Per H. Svensson, ${ }^{\text {ab }}$ Minh Tam Hoang, ${ }^{c}$ Ilwhan Oh, ${ }^{c}$ Lars Kloo ${ }^{a}$ \\ and James M. Gardner*a
}

The synthetic route and properties of three 2D hybrid organic/inorganic lead iodide perovskite materials are reported. The $2 \mathrm{D}$ perovskites were synthesized from the reaction between $\mathrm{Pbl}_{2}$ and the di-cations of 1,4diaminobutane, 1,6-diaminohexane, and 1,8-diaminooctane. The resulting products were $\left[\mathrm{NH}_{3}\left(\mathrm{CH}_{2}\right)_{4} \mathrm{NH}_{3}\right]$ $\left.\mathrm{Pbl}_{4}\left(\mathrm{BdAPbl}_{4}\right),\left[\mathrm{NH}_{3}\left(\mathrm{CH}_{2}\right)_{6} \mathrm{NH}_{3}\right] \mathrm{Pbl}_{4}(\mathrm{HdAPbl})_{4}\right)$, and $\left.\left[\mathrm{NH}_{3}\left(\mathrm{CH}_{2}\right)_{8} \mathrm{NH}_{3}\right] \mathrm{Pbl}_{4}(\mathrm{OdAPb})_{4}\right)$. Structural characterization shows that two dimensional perovskite structures were formed with inorganic structural planes separated by organic layers. Absorption spectra show band gaps of $\left.2.37 \mathrm{eV}\left(\mathrm{BdAPbl}_{4}\right), 2.44 \mathrm{eV}(\mathrm{HdAPbl})_{4}\right)$, and $2.55 \mathrm{eV}$ $(\mathrm{OdAPbl})_{4}$. The 2D perovskite materials were investigated as light absorbing materials in solid state solar cells. The best performing material under moist, ambient conditions was $\mathrm{BdAPbl}_{4}(1.08 \%$ efficiency), which was comparable to methylammonium $\mathrm{Pb}(\|)$ iodide $\left(\mathrm{MAPb}_{3}\right)$ solar cells (2.1\% efficiency) manufactured and studied under analogous conditions. When compared to $\mathrm{MAPb}_{3}$, the 2D materials have larger band gaps and lower photoconductivity, while $\mathrm{BdAPbl}_{4}$ based solar cells shows a comparable absorbed photon-to-current efficiency as compared to $\mathrm{MAPbl}_{3}$ based ones.

\section{Introduction}

Methylammonium lead(II) halide perovskite materials have emerged as unexpectedly promising new materials for solar energy conversion. ${ }^{1-8}$ The first use of methylammonium lead iodide $\left(\mathbf{M A P b I}_{3}\right)$ in solar cells was reported in 2009 by Miyasaka et al., ${ }^{1}$ who used $\mathbf{M A P b I}_{3}$ as a sensitizer in a liquid junction solar cell where it produced a power conversion efficiency of $3.8 \%$. The low stability of $\mathbf{M A P b I}_{3}$ in contact with the liquid electrolyte was a clear problem; however, by introducing these materials in solid-state solar cells rapid improvements in efficiency were reported. ${ }^{3-8}$ Through substantial engineering and optimization, efficiencies in excess of $20 \%$ (up to $22.1 \%$ as certified by NREL ${ }^{9}$ ) have been obtained, with many labs routinely reporting efficiencies of $17-18 \% .^{\mathbf{1 0 , 1 1}}$ While the improvements in efficiency

\footnotetext{
${ }^{a}$ Applied Physical Chemistry, Department of Chemistry, KTH Royal Institute of Technology, SE-100 44, Stockholm, Sweden. E-mail:jgardner@kth.se

${ }^{b}$ SP Process Development, Forskargatan, 15121 Södertälje, Sweden

${ }^{c}$ Department of Applied Chemistry, Kumoh National Institute of Technology, Gumi, Gyeongbuk, Korea

$\dagger$ Electronic supplementary information (ESI) available: Tables S1-S4 including atomic parameters of the structure of the three new materials, Fig. S1 and S2, two 2D structures of butyl 1,4-diammonium lead iodide and octyl 1,8-diammonium lead iodide along the three crystallographic axes. Tables S5-S11 and Fig. S3-S9 describe structural and photochemical characterization. CIF files for the structures are available at the Cambridge Crystallographic Data Center (CCDC) referring to the deposition numbers 1420433 and 1420434 for

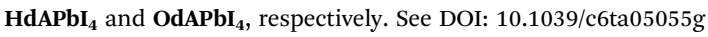

have been staggeringly fast, an underlying understanding of what physical and chemical properties make $\mathbf{M A P b I}_{3}$ so efficient in solar cells is lacking. Some effort has been focused on investigating the relationship between the structure and the emergent physical properties for the perovskite materials. ${ }^{\mathbf{1 2 - 1 7}}$ The crystal structure of $\mathbf{M A P b I}_{\mathbf{3}}$ shows a three dimensional (3D) network of corner-sharing $\left[\mathrm{PbI}_{6}\right]$ structural units in a perovskite type structure, which has randomly oriented methylammonium cations filling the voids between the corner-linked $\left[\mathrm{PbI}_{6}\right]$ octahedra. ${ }^{18,19}$ The atomic positions in the methylammonium cation, which has not been resolved crystallographically due to rapid scrambling, may play a role in the charge-separation and exciton dynamics. ${ }^{20,21}$ However, high efficiency solar cells from the structurally and electronically similar material $\mathrm{CsSnI}_{3}$ suggest that the cation may not have a significant role in these processes. The perovskite material combines a number of impressive physical properties including a medium direct band gap ${ }^{\mathbf{1 8 , 1 9}}$ high extinction coefficient, ${ }^{22}$ low exciton binding energy, ${ }^{22,23}$ and high photoconductivity. ${ }^{19,22}$ In addition, facile solution processing of this family of materials makes them an interesting candidate for use as light absorber in solar cells. Laboratory solar cells have shown promising efficiencies, but there are several major issues that appear to be fundamental to the material itself. Current experimental results show that the methylammonium lead iodide layer is not stable under ambient conditions. ${ }^{24}$ One possible reason could be dissolution of the organic cation and iodide by water from the ambient moisture in air, which advances the decomposition of $\mathbf{M A P b I}{ }_{3}$ to residual $\mathbf{P b I}_{2}$. Furthermore, thermal 
instability of the methylammonium lead iodide adds to faster degradation of the materials and hence to degradation of the solar cell performance. ${ }^{24,25}$ Thermal instability of $\mathbf{M A P b I}_{3}$ results from a tetragonal-to-cubic phase transition below $60{ }^{\circ} \mathrm{C}$ (ref. 15) and a faster degradation of the material. ${ }^{18,26}$ As well, the band gap for $\mathbf{M A P b I}_{3}$ is larger than the ideal one for a single junction solar cell and too small to couple to most other known solar cell materials in tandem devices. Previous reports have discussed how the structural properties of these materials influence physical properties. ${ }^{15}$ In our previous work, we have shown that an increase of the size of cation leads to an abrupt change in dimensionality of the alkylammonium lead(II) iodide structure and the resulting physical properties. ${ }^{19}$ For the formamidinium lead(II) iodide perovskite, the same effect has been observed, where three dimensional networks exhibit different properties as compared to one dimensional ones. ${ }^{27}$ Layered 2D perovskite structures of hybrid organic-inorganic materials were studied more than a decade ago based on $\mathrm{Pb}^{2+}, \mathrm{Bi}^{3+}$, and $\mathrm{Sn}^{2+} \cdot{ }^{28-30}$ These 2D layered perovskites can be synthesized following similar solution based synthetic strategies as used for $\mathbf{M A P b I}_{3} \cdot{ }^{31,32}$ For $\mathrm{Pb}^{2+}$ the pure 2D perovskite has the general composition of $\mathrm{A}_{2} \mathrm{PbX}_{4} ; \mathrm{A}$ is an organic cation and $\mathrm{X}$ is a halide anion. There have been previous reports on layered perovskites based on mixed cations of methylammonium together with large organic cations. ${ }^{11,28,32}$ These materials maintain structures characterized by multiple layers of $\mathbf{M A P b I}_{3}$, just like a 3D perovskite, interrupted by large cations that promote the formation of $2 \mathrm{D}$ perovskite monolayers. ${ }^{31,32}$ Crystallographic studies show that these monolayers contain immobile cations, in strong contrast to the methylammonium cation in $\mathbf{M A P b I} \mathbf{I}_{3}$ layers. We are unaware of any studies that have examined the photoelectric properties of $2 \mathrm{D}$ perovskites with dialkylammonium cations. There are strong motives to examine $2 \mathrm{D}$ perovskite materials, since they are expected to exhibit similar physical properties as the $3 \mathrm{D} \mathbf{M A P b I}_{3}$ perovskites but can accommodate a wider variety of cations and thus should obviate stability issues present in $\mathbf{M A P b I}_{3}$.

In this work, we report on three layered 2D perovskite materials with substantial moisture and thermal stability. We have varied the length of the organic chain in the diammonium cations employed to promote two dimensional structures. Subsequently we have investigated the physical properties of the materials obtained, and lastly the materials have been employed in solar cells. Layered structures for the material have been addressed as one possible strategy to escape the low moisture resistance of perovskite solar cells. ${ }^{29,30,32}$ Further studies have been followed by Cao et al. indicating a capability for two dimensional structure to overcome the stability issue. ${ }^{31}$

\section{Experimental section}

Chemicals were purchased from Sigma-Aldrich unless otherwise stated. Spiro-OMeTAD ((2,20,7,70-tetrakis[N,N-di(-4-methoxyphenyl)amino)-9,9'-spirobifluorene, purity $\geq 99.5$ HPLC) was purchased from Xi'an Polymer Light Technology Corp. Dyesol (18NRT) $\mathrm{TiO}_{2}$ paste was used. Hydriodic acid was purchased from Alfa Aesar, $57 \%$ w/w aqueous solution stabilized with $1.5 \%$ hypophosphorous acid.

\section{Synthesis}

In order to prepare the diammonium iodide salts, $1 \mathrm{~mol}$ equivalent of 1,4-diaminobutane (99\%), 1,6-diaminohexane (98\%), or 1,8-diaminooctane (98\%) was mixed with $2 \mathrm{~mol}$ equivalents of hydriodic acid solution followed by stirring for 2 hours at $0{ }^{\circ} \mathrm{C}$ (on an ice bath). The diammonium iodide salts were recovered by evaporation of the solvent and washing with diethyl ether $(\geq 99.0 \%)$.

Lead iodide (99.999\%-2 mol equivalents) was dissolved in $4 \mathrm{~mL}$ HI solution. Diammonium iodide salts ( $1 \mathrm{~mol}$ equivalent) were dissolved in $3 \mathrm{~mL}$ excess $\mathrm{HI}$. The two solutions were mixed and stirred at $90{ }^{\circ} \mathrm{C}$ for 1 hour. In order to grow single crystals of the materials, after half of the solvent is evaporated, the magnetic stir bar was taken out from solution and the temperature was gradually decreased $\left(5{ }^{\circ} \mathrm{C}\right.$ per hour) to $-10{ }^{\circ} \mathrm{C}$. Crystals were recovered from the solution and used for the crystallographic investigations. Residual precipitation was washed with diethyl ether and used for the other methods of characterization.

\section{Solar cells fabrication}

Fluorine-doped tin-oxide (FTO) coated glass substrates (Pilkington TEC15) were cleaned by detergent, ethanol and acetone, as reported previously. ${ }^{19}$ Oxygen plasma cleaning was used to remove remaining organic traces. A solution of titanium isopropoxide in ethanol and hydrochloric acid (mixture of 175 $\mu \mathrm{L}$ of titanium isopropoxide in $1.25 \mathrm{ml}$ ethanol and $175 \mu \mathrm{L}$ of 2 $\mathrm{M} \mathrm{HCl}$ in $1.25 \mathrm{~mL}$ ethanol) ${ }^{24}$ was used for preparing a compact $\mathrm{TiO}_{2}$ layer ( $\sim 100 \mathrm{~nm}$ thick). A compact blocking layer was prepared through spin coating of the titanium isopropoxide solution onto the FTO glass substrate at $2000 \mathrm{rpm}$ for 30 seconds. The film was then sintered at $520{ }^{\circ} \mathrm{C}$ for 30 minutes. A mixture of $1: 5$ wt ratio of $\mathrm{TiO}_{2}$ Dyesol paste with isopropanol was used to spin coat a layer of $\mathrm{TiO}_{2}$ on top of the blocking layer. Sintering at $500{ }^{\circ} \mathrm{C}$ for $30 \mathrm{~min}$ was performed to produce a mesoporous $\mathrm{TiO}_{2}$ layer. This was followed by a $\mathrm{TiCl}_{4}$ treatment by immersing the sintered film into a $40 \mathrm{mM}$ solution of $\mathrm{TiCl}_{4}$ in water at $70^{\circ} \mathrm{C}$ for 30 minutes. After rinsing with deionized water and ethanol, the films were sintered for the last time at $500{ }^{\circ} \mathrm{C}$ for 30 minutes.

Dimethylformamide (DMF; purity $\geq 99 \%$ ) precursor solutions were spin coated onto the $\mathrm{TiO}_{2}$ films and then the films were annealed at $90{ }^{\circ} \mathrm{C}$ for $10 \mathrm{~min}$. The hole transport material (HTM) solution was made of $80 \mathrm{mM}$ SpiroOMeTAD, $200 \mathrm{mM}$ 4-tert-butylpyridine (TBP, 99\%) and $30 \mathrm{mM}$ bis(trifluoromethane) sulfonimide lithium salt (LiTFSI, 99\%, Io-li-tec). After cooling the film to room temperature from annealing, the HTM solution was spin coated onto the substrate at $2000 \mathrm{rpm}$ for 30 seconds. In the last step silver thermal evaporation was performed to generate a $200 \mathrm{~nm}$ silver metal layer on top of the HTM layer as counter electrode. ${ }^{3,5,19}$ The whole process of the solar cell fabrication was performed in ambient air and at ambient temperature, except for the silver evaporation process. The laboratory temperature was $23.4{ }^{\circ} \mathrm{C}$ with a humidity of $55.6 \%$ as measured by an AMPROBE TH-3 hygrometer (a typical summer day in Stockholm, Sweden). 


\section{Characterization techniques}

Absorption spectroscopy. Spin coating of a $\mathrm{TiO}_{2}$ solution with ratio of $1 \mathrm{TiO}_{2}$ Dyesol paste: 3.5 ethanol (99.5\%) produced $\mathrm{a} \sim 250 \mathrm{~nm}$ mesoporous film on a microscopic slide. After sintering the film, it was used as substrate for the UV-visible absorption measurements. DMF solutions of the materials were spin coated onto the $\mathrm{TiO}_{2}$ films. This was followed by heating at $90{ }^{\circ} \mathrm{C}$ for $10 \mathrm{~min}$. UV-visible absorption spectra were recorded using UV-visible Cary 300 spectrophotometer.

Single crystal X-ray crystallography. Crystals were selected for study and mounted on a cryoloop using a low-temperature immersion oil and placed in an $\mathrm{N}_{2}$ cold stream. Single-crystal X-ray data were collected on a Bruker APEXII diffractometer (MoK $\alpha$ radiation), equipped with a CCD detector, at $200 \mathrm{~K}$. The data sets were recorded with $\omega$-scans and $\varphi$-scans, and integrated with the Bruker SAINT ${ }^{33}$ software package. The absorption correction (Bruker SADABS ${ }^{33}$ ) was based on the fitting of a function to the empirical transmission surface as sampled by multiple equivalent measurements. Solution and refinement of the crystal structures were carried out using SHELXS and SHELX within the Bruker program package. ${ }^{33}$ Structure solution by direct methods resolved positions of all atoms except hydrogens. The remaining non-hydrogen atoms were located by alternating cycles of least-squares refinements and difference Fourier maps. Hydrogen atoms were placed at calculated positions. The final refinements were performed with anisotropic thermal parameters for all non-hydrogen atoms. A summary of pertinent information relating to unit cell parameters, data collection, and refinement is provided in Tables S1-S4. $\dagger$

Thermal analysis/differential scanning calorimetry (DSC). The DSC analyses were performed on a Mettler DSC822e, equipped with a Thermo Haake EK45/MT cooler. The samples were weighted into $40 \mu \mathrm{L} \mathrm{Al-cups,} \mathrm{which} \mathrm{were} \mathrm{then} \mathrm{closed} \mathrm{with}$ pierced lids. Each sample was scanned from 25 to $500{ }^{\circ} \mathrm{C}$, with a scan rate of $10^{\circ} \mathrm{C}$ per minute using $80 \mathrm{ml}$ nitrogen per minute as purge gas.

X-ray powder diffraction. Well ground powders of the samples were used for collecting powder X-ray diffraction patterns. A PANalytical-X'Pert PRO diffractometer employing $\mathrm{Cu}$-Ka radiation was used for scanning over a $2 \theta$ range of $10^{\circ}$ to $40^{\circ}$.

Conductivity. The measurement was carried by an experimental procedure described in previous work. ${ }^{19}$ Briefly, precursor solutions of the materials were spin coated onto a $\mathrm{TiO}_{2}$ film (on a non-conductive substrate). This was followed by evaporation of a silver metal layer as back contact. The current response under different bias voltages was collected under light illumination. The obtained slope of the currentvoltage curve was used for calculation of the conductivity by using eqn (1).

$$
\sigma=\frac{W}{R L d}
$$

where $W$ is the width between the silver metal contacts, $d$ is the thickness of the perovskite/ $/ \mathrm{TiO}_{2}$ film corrected for the $\mathrm{TiO}_{2}$ volume, and $L$ is the length of the silver metal film. The $\mathrm{TiO}_{2}$ films have $60 \%$ porosity. To correct for the volume of the $\mathrm{TiO}_{2}$, the thickness of the materials $(d)$ was multiplied by $60 \%$.

Cyclic voltammetry (CV). In order to prepare samples for electrochemical and UPS measurements, $0.8 \mathrm{M}$ perovskite solution in DMF (stirred at $60{ }^{\circ} \mathrm{C}$ overnight) was spin coated onto a cleaned FTO glass substrate followed by heating at $100{ }^{\circ} \mathrm{C}$ for $10 \mathrm{~min}$. Then the coated substrate was adopted as working electrode in a Teflon cell with a Pt mesh counter electrode and an $\mathrm{Ag} / \mathrm{AgCl}$ quasi-reference electrode (QRE). The inner solution in the QRE was $10 \mathrm{mM} \mathrm{AgNO}+0.1 \mathrm{M}$ tetrabutylammonium perchlorate (TBAP; 99\%, Sigma Aldrich) in acetonitrile (99.7\%) and the QRE's potential was calibrated to be $0.537 \mathrm{~V} v s$. the Standard Hydrogen Electrode (SHE) by using the ferrocene redox system as reference. The supporting electrolyte employed was 0.1 M TBAP in acetonitrile and the cyclic voltammograms were recorded using a potentiostat (model SP-150; BioLogic Science Instruments).

Ultraviolet photoelectron spectroscopy (UPS). The kinetic energy was obtained with $0.05 \mathrm{eV}$ resolution using a photoelectron spectrometer with a UV intensity of $10 \mathrm{nW}$ (model Ac-2; Hitachi).

Device characterization. The current-voltage $(I-V)$ characteristics of the solar cells were obtained using an external potential bias. The generated photocurrent was recorded with a Keithley model 2400 digital source meter. The light source was a $300 \mathrm{~W}$ collimated xenon lamp (Newport). A certified silicon solar cell (Fraunhofer ISE) was used for calibration of the light source. The light intensity used was $100 \mathrm{~mW} \mathrm{~cm}^{-2}$ at AM $1.5 \mathrm{G}$ solar light conditions. The incident photon-to-current efficiency (IPCE) spectra were obtained on a computer-controlled setup. This setup is an assembly of a xenon lamp (Spectral Products ASB-XE-175), a monochromator (Spectral Products CM110) and a Keithley digital multimeter (Model 2700). The setup was calibrated with a certified silicon solar cell (Fraunhofer ISE) prior to characterization. Solar cells were masked during the measurement with an aperture area of $0.126 \mathrm{~cm}^{2}$ exposed under illumination.

\section{Results and discussion}

\section{X-ray diffraction}

High quality single crystals of $\mathbf{H d A P b I}_{4}$ and $\mathbf{O d A P b I} \mathbf{4}_{\mathbf{4}}$ were obtained through the synthetic procedure (described in the Experimental) with no detectable impurities. The obtained crystals were subjected to X-ray diffraction studies at $200 \mathrm{~K}$. Details of the data collection and other crystallographic information for the structures are given in Tables S2-S4, $\uparrow$ and important bond lengths and bond angles are provided in Table S1.†

Due to disorder effects in the $\mathbf{B d A P b I}{ }_{4}$ structure, the crystal structure proposed should only be regarded as a structural model. Powder diffraction X-ray characterization was performed on bulk powders of the materials and was compared to the calculated powder diffraction patterns obtained from single crystal data. The calculated and experimentally obtained diffraction patterns match, implying that the single crystal structures are representative for the bulk of the materials. The 

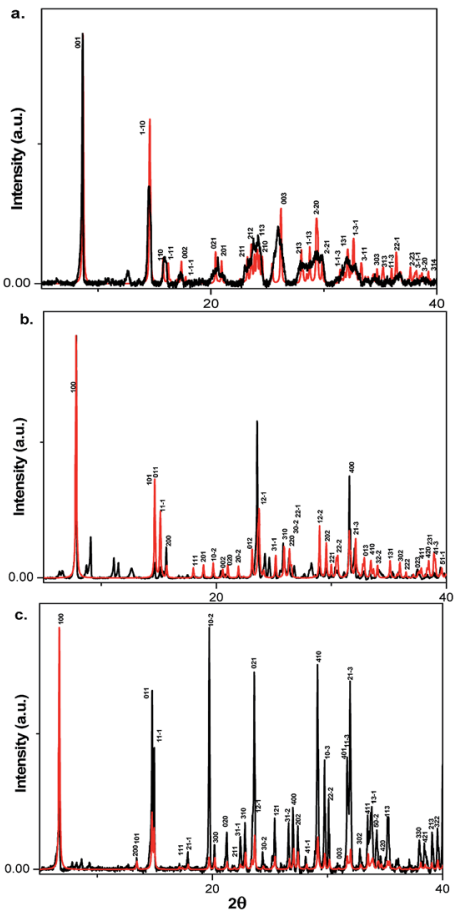

Fig. 1 --- X-ray powder diffraction and --- calculated patterns from single crystal data of (a) $\mathrm{BdAPbl}_{4}$ (b) $\mathrm{HdAPbl}_{4}$ and (c) OdAPbl 4 .

diffractograms with Miller indices generated from single crystal data are depicted in Fig. 1.

Broad peaks can be noted in the diffractograms of $\mathbf{B d A P b I}_{\mathbf{4}}$. The reason can most likely be attributed to disorder in the structure. Deviations in relative peak intensities noted between calculated and experimentally determined diffractograms are likely to be caused by crystal orientation bias in the powders studied. For instance, this effect refers to the (100) plane in all three materials. X-ray diffraction pattern of the material's thin film on $\mathrm{TiO}_{2}$ layer showed that for $\mathbf{B d A P b I}{ }_{4}$ and $\mathbf{H d A P b I} \mathbf{A}_{4}$ films are representative of the bulk materials, while for OdAPbI some new peaks are recorded for the thin films materials on the $\mathrm{TiO}_{2}$. This mismatched pattern can be originated from the bigger unit cell volume for $\mathbf{O d A P b I}_{4}$ and the related interference for material crystallization on mesoporous $\mathrm{TiO}_{2}$ (see Fig. S6 $\dagger$ ). Spin coated $\mathbf{M A P b I}_{3}$ films from DMF solutions were investigated by powder X-ray diffraction and the diffractograms are presented in Fig. S7. $\dagger$ In accordance to the previous reports, ${ }^{24}$ $\mathrm{PbI}_{2}$ peaks were obtained indicating a small portion of $\mathrm{PbI}_{2}$ due to instability of $\mathbf{M A P b I}_{3}$ in the ambient atmosphere.

All three materials show very related two dimensional (2D) perovskite structures consisting of planes of corner-sharing octahedra of $\left[\mathrm{PbI}_{6}\right]$ units separated by the organic cation (Fig. 2, 3 , S1 and S2 $\dagger$ ). A representative structure of $2 \mathrm{D} \mathbf{B d A P b I}_{4}$ is presented in Fig. S1, $\dagger$ 2D HdAPbI ${ }_{4}$ and $2 \mathrm{D}$ OdAPbI ${ }_{4}$ are depicted in Fig. 2 and $\mathrm{S} 2, \dagger$ respectively.

$\mathbf{B d A P b I}_{\mathbf{4}}$ crystallizes in the triclinic system in the space group $P 1$ and with the lattice parameters $a=8.4815(14) \AA, b=$ 8.8472(14) $\AA$, and $c=11.2028(17) \AA$.

Both $\mathbf{H d A P b I}_{4}$ and $\mathbf{O d A P b I}_{4}$ crystallize in the monoclinic system in the $P 2_{1} / c$ space group symmetry and in a slightly larger

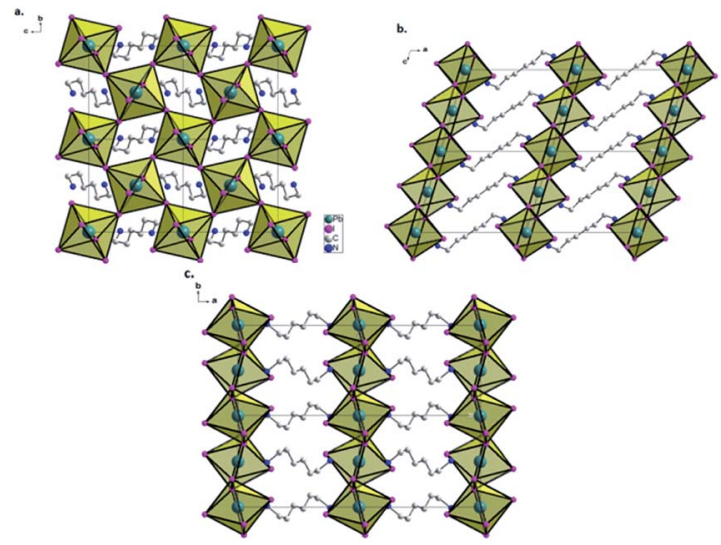

Fig. 2 Structure of $\mathrm{HdAPbl}_{4}$ showed along the three crystallographic axes.

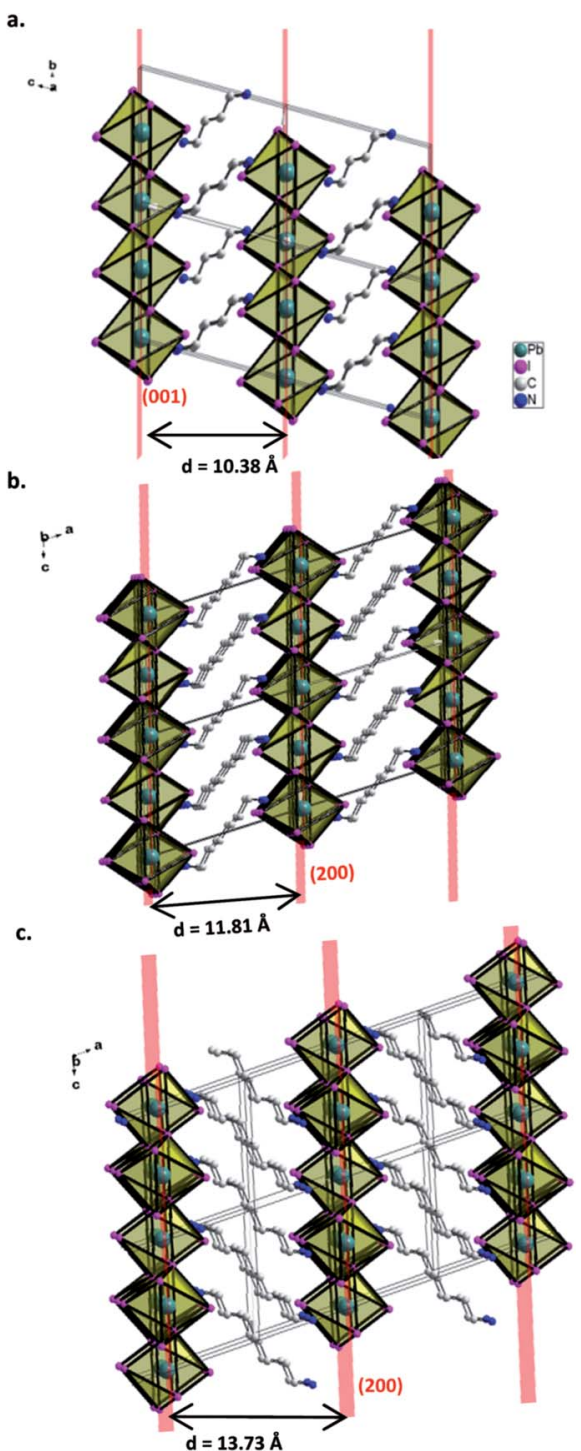

Fig. 3 Structural planes of (a) $\mathrm{BdAPbl}_{4}$ (b) $\mathrm{HdAPbl}_{4}$ (c) OdAPbl ${ }_{4}$. 
unit cell for OdAPbI $\mathbf{H}_{4}$. The unit cell of $\mathbf{H d A P b I}_{\mathbf{4}}$ is described by $a=11.8055(6) \AA, b=8.4509(4) \AA$, and $c=9.0262(5) \AA$, and for OdAPbI $_{4}$ by $a=13.7343(10) \AA, b=8.3435(5) \AA$, and $c=$ 9.0041(6) A. More details of the crystallographic analyses are presented in Table 1 . The organic cations are imbedded in the structure as a link between the lead iodide planes. Hence, the distance between the inorganic planes correlates with the size of the cation used. For $\mathbf{B d A P b I}$, the inorganic component of the structure is confined along the (001) planes connected by the BdA cations. The distance between the planes (defined as the distance between the $\mathrm{Pb}$ atoms in adjacent planes) is $10.4 \AA$. As could be expected, this distance is larger in $\mathbf{H d A P b I} \mathbf{I}_{\mathbf{4}}$ and $\mathbf{O d A P b I} \mathbf{I}_{\mathbf{4}}$ due to the larger organic cations present in the structures. The $2 \mathrm{D}$ perovskite planes in $\mathbf{H d A P b I}_{4}$ follow the (200) direction with a $11.8 \AA$ interplane distance. This trend is extended by $\mathbf{O d A P b I}_{4}$ with an interplane $\mathrm{Pb}-\mathrm{Pb}$ distance of $13.7 \AA$. The interlayer distances between iodide ions in two adjacent planes are $4.25 \AA$ in $\mathbf{B d A P b I}_{4}, 6.06 \AA$ in $\mathbf{H d A P b I}_{4}$, and $8.29 \AA$ in $\mathbf{O d A P b I}_{4}$.

The distances between two central $\mathrm{Pb}$ octahedral atoms inside a plane are 6.10, 6.19 and $6.14 \AA$ for $\mathbf{B d A P b I}{ }_{4}, \mathbf{H d A P b I}_{4}$ and $\mathrm{OdAPbI}_{4}$, respectively. These are comparable to the corresponding $6.28 \AA$ distances found in $\mathbf{M A P b I}_{3}$. It is notable that the intraplane arrangement of the corner-sharing $\left[\mathrm{PbI}_{6}\right]$ octahedra is considerably less ordered with $\mathrm{Pb}-\mathrm{I}-\mathrm{Pb}$ angles of $150^{\circ}$, $148^{\circ}$ and $147^{\circ}$ for $\mathbf{B d A P b I}_{4}, \mathbf{H d A P b I}_{4}$, and $\mathbf{O d A P b I}_{4}$, respectively, in contrast to the $180^{\circ}$ angle found in the cubic $\mathbf{M A P b I} \mathbf{I}_{3}$ structure. ${ }^{19}$ More details are presented in Table $\mathrm{S} 1 \dagger$ together with structural details of methylammonium lead iodide in Table 1.

The well-defined cation positions obtained from the crystallography in the 2D materials indicate that cation movement and migration must be considerably lower than in $\mathbf{M A P b I}_{3}$. The immobile cations will generate a permanent dipole moment throughout the material network rather than the rotating dipole moment in $\mathbf{M A P b I}_{3}$, which may affect the process of charge separation. $^{20,21}$

\section{Thermal analysis}

The thermal stability of the new materials and examination of possible phase transitions were investigated using Differential Scanning Calorimetry (DSC). An impressive stability was observed for the materials up to at least $205{ }^{\circ} \mathrm{C}$ for $\mathbf{B d A P b I}{ }_{4}$ and $210{ }^{\circ} \mathrm{C}$ for $\mathbf{H d A P b I}_{4}$ (Fig. S4 and $\mathrm{S} 5 \dagger$ ). No phase transition was observed up to these temperatures indicating high thermal stability for these materials in contrast to $\mathbf{M A P b I}_{3}$.

\section{Absorption spectroscopy}

The optical properties of the materials were investigated by UV-visible characterization and the results are presented in Fig. 4. The strong absorption at longer wavelengths can be attributed to transitions over the band gap of the materials. Band gaps were estimated using the Tauc formula ${ }^{34}$ (Tauc plots are presented in Fig. S3†). The band edges emerge as $2.37 \mathrm{eV}$, $2.44 \mathrm{eV}$ and $2.55 \mathrm{eV}$ for $\mathbf{B d A P b I}_{4}, \mathbf{H d A P b I}_{4}$, and $\mathrm{OdAPbI}_{4}$, respectively. The increase in structural spacing between the inorganic planes in the materials can clearly be correlated to an increase in the band gaps. This may be related to quantum confinement reducing orbital overlap between the lead iodide structural planes and hence a shift of the band gap to higher energies. Although band edges are not very different, incorporation of a bigger cation drastically decrease the slope of the absorption edge, thus suggesting a trend in decreasing absorption coefficient. Previous studies have shown different optical properties for different crystal sizes of the 3D lead perovskites. ${ }^{35,36}$ Assuming a similar behavior for this class of $2 \mathrm{D}$ perovskites, due to the larger volume of the cation in $\mathbf{O d A P b I}_{4}$ the crystallinity of the material may be lower, leading to a lower

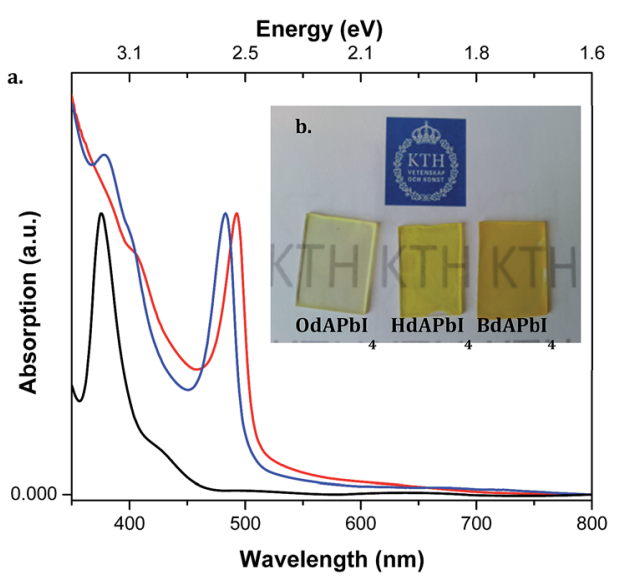

Fig. 4 (a) UV-visible absorption spectra of --- $\mathrm{BdAPbl}_{4},---\mathrm{HdAPbl}_{4}$, and --- OdAPbl 4 on spin-coated mesoporous thin films of $\mathrm{TiO}_{2}$ (b) photographs of the materials.

Table 1 Structural details for three 2D perovskite materials compared with the 3D $\mathrm{MAPbl}_{3}$ (ref. 19)

\begin{tabular}{|c|c|c|c|c|}
\hline & $\mathbf{B d A P b I}_{4}$ & $\mathrm{HdAPbI}_{4}$ & $\mathrm{OdAPbI}_{4}$ & $\mathbf{M A P b I}_{3}$ (ref. 19) \\
\hline Space group & $P 1$ (1) (triclinic) & $P 21 / c$ (monoclinic) & $P 21 / c$ (monoclinic) & $P m \overline{3} m$ \\
\hline \multirow[t]{3}{*}{ Cell lengths $(\AA)$} & $a 8.4815(14)$ & $a 11.8055(6)$ & a $13.7343(10)$ & $a 6.284$ \\
\hline & b $8.8472(14)$ & $b 8.4509(4)$ & $b 8.3435(5)$ & $b 6.284$ \\
\hline & c 11.2028(17) & $c 9.0262(5)$ & $c 9.0041(6)$ & $c 6.284$ \\
\hline \multirow[t]{3}{*}{ Cell angles } & $\alpha=76.83(7)$ & $\alpha 90.00$ & $\alpha 90.00$ & $\alpha 90.00$ \\
\hline & $\beta=69.67$ (7) & $\beta 107.073(2)$ & $\beta$ 106.577(4) & $\beta 90.00$ \\
\hline & $\gamma=89.46(9)$ & $\gamma 90.00$ & $\gamma 90.00$ & $\gamma 90.00$ \\
\hline Cell volume $\left(\AA^{3}\right)$ & 765.28 & 860.833 & 988.915 & 248.15 \\
\hline$Z$ & 4 & 4 & 4 & 1 \\
\hline
\end{tabular}


absorption coefficient. The absorption edges observed correlate very well with the IPCE spectra of the resulting solar cells (see Fig. 6c).

\section{Cyclic voltammetry and ultraviolet photoelectron spectroscopy (UPS)}

The band energy diagram provides deeper insights into the charge transfer dynamics of the solar cells constructed based on the $2 \mathrm{D}$ perovskite materials (Fig. 5). Due to the high stability of these materials, valence band maxima were determined by cyclic voltammetry (CV). In addition Ultraviolet Photoelectron Spectroscopy (UPS) was used to confirm the CV results. Results from the two types of experiments match well (Table S9†). The UPS results suggest direct band gap behavior for the three materials. The $\mathrm{CV}$ results offer valence band positions at $-5.33 \mathrm{eV},-5.56 \mathrm{eV}$, and $-5.36 \mathrm{eV}$ for $\mathbf{B d A P b I}_{4}, \mathbf{H d A P b I}_{4}$, and OdAPbI $_{4}$, respectively.

It is notable that the position of the valence band edge energy does not change drastically (less than $0.16 \mathrm{eV}$ ) with respect to the structural changes from $3 \mathrm{D}$ to $2 \mathrm{D}\left(c f . \mathbf{M A P b I}_{3}\right)$. However the conduction band edge energy increases upon a decrease in dimensionality. This suggests that the comparatively low energy of the $\mathrm{TiO}_{2}$ conduction band may limit the obtained open circuit voltages from these materials.

For the 3D perovskite $\mathbf{M A P b I}_{3}$, previous studies have shown a large contribution from iodide p-orbitals to the valence band, while the conduction band mostly consists of contributions from the $\mathrm{Pb}$ p-orbital. ${ }^{37,38}$ The above results indicate that the composition of the valence bands in the new 2D materials should be similar to the $3 \mathrm{D}$ compound, but that the orbital composition of the conduction bands may differ.

\section{Conductivity}

Conductivities of the materials were obtained as $1.3 \times 10^{-5} \mathrm{~S}$ $\mathrm{cm}^{-1}, 1.3 \times 10^{-5} \mathrm{~S} \mathrm{~cm}^{-1}$, and $1.3 \times 10^{-5} \mathrm{~S} \mathrm{~cm}^{-1}$ for BdAPbI $\mathbf{I}_{4}$, $\mathbf{H d A P b I}_{4}$, and $\operatorname{OdAPbI}_{4}$, respectively, where $\mathbf{M A P b I}_{3}$ was employed as a control in the measurements. The conductivities

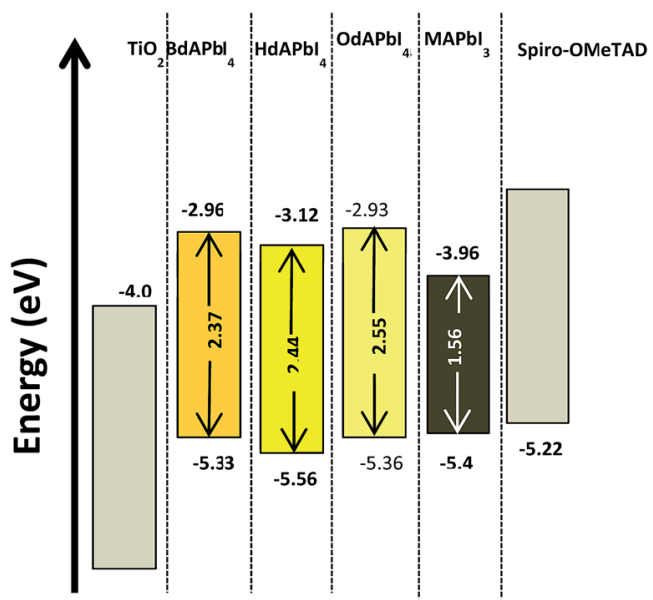

Fig. 5 Band energy diagrams of the 2D perovskite materials compared to other components of the solar cells. decrease one order of magnitude going from 3D to 2D structures. In our previous study, ${ }^{19}$ we showed that the conductivity is dependent on the dimensionality of the structure of the materials, where the conductivity of 3D $\mathbf{M A P b I}_{3}$ was $1.1 \times 10^{-4}$ $\mathrm{S} \mathrm{cm}{ }^{-1}$, while a conductivity of $1.3 \times 10^{-6} \mathrm{~S} \mathrm{~cm}^{-1}$ was recorded for the $1 \mathrm{D}\left(\mathrm{CH}_{3} \mathrm{CH}_{2} \mathrm{NH}_{3}\right) \mathrm{PbI}_{3}$.

\section{Solar cells}

Solid state solar cells were prepared based on the three new 2D materials as the light absorbing/charge transport component. Precursor solutions of the three 2D materials were spin-coated into $\mathrm{TiO}_{2}$ mesoporous films (Fig. 6a). Solar cells based on the $\mathbf{M A P b I}_{3}$ material were prepared under the same conditions for comparison (well aware of the fact the moist conditions will produce $\mathbf{M A P b I}_{3}$-based solar cells of quite poor quality). The better moisture resistance of the $2 \mathrm{D}$ materials was indeed a beneficial property in the production of robust and easy to handle solar cells. The best solar cells based on $\mathbf{B d A P b I} \mathbf{I}_{\mathbf{4}}$ showed an efficiency of $1.08 \%$ with $2.9 \mathrm{~mA} \mathrm{~cm}^{-2}$ in current density. To the best of our knowledge, this represents the highest efficiency obtained from any pure 2D perovskite solar cell. ${ }^{31}$ Considering how much light is lost because of the relatively large band gap for the material $(2.37 \mathrm{eV})$ and losses due to thermalization for electrons injected into $\mathrm{TiO}_{2}$, the results are rather impressive. For the solar cells based on $\mathbf{B d A P b I}{ }_{4}, \mathbf{H d A P b I}_{4}$ and $\mathbf{O d A P b I} \mathbf{I}_{4}$, the open circuit voltages were $870 \mathrm{mV}, 725 \mathrm{mV}$ and $732 \mathrm{mV}$ respectively. Lower voltages were obtained for some of the $2 \mathrm{D}$ based solar cells as compared to $805 \mathrm{mV}$ obtained for the 3D $\mathbf{M A P b I}_{3}$ based solar cells. Substantial charge recombination losses in $\mathbf{O d A P b I} \mathbf{A}_{\mathbf{4}}$ and $\mathbf{H d A P b I} \mathbf{H}_{\mathbf{4}}$ solar cells likely reduced the difference in quasi-Fermi levels for $\mathrm{TiO}_{2}$ and Spiro-OMeTAD, decreasing the open-circuit voltage for these devices. ${ }^{39}$ The low performance of the $\mathbf{M A P b I}_{3}$ solar cells as compared to the previously published reports ${ }^{3}$ may be attributed to the poor stability of the $\mathbf{M A P b I}_{3}$ films under humid conditions. ${ }^{31}$ It is notable that the pure iodide methylammonium lead iodide

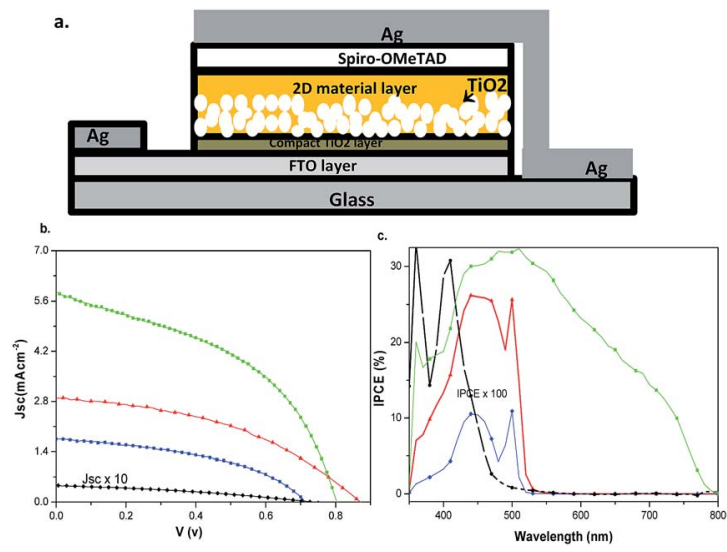

Fig. 6 (a) Schematic device architecture for the mesoporous solid state solar cells, (b) I-V and (c) IPCE curves of the solar cells based on --- $\mathrm{BdAPbl}_{4},---\mathrm{HdAPbl}_{4},--\mathrm{OdAPbl}_{4}$, and --- MAPbl${ }_{3}$. I-V characterizations were performed under 1 sun AM1.5G illumination. For ease of comparison the $\mathrm{OdAPbl}_{4}$ based solar cell results, the $J_{\mathrm{sc}}$ is multiplied by a factor of 10 and the IPCE is multiplied by a factor of 100 
Table 2 Photovoltaic performance of the fabricated solar cells

\begin{tabular}{llllllll}
\hline & Eff $(\%)$ & $V_{\text {oc }}(\mathrm{V})$ & $J_{\mathrm{sc}}\left(\mathrm{mA} \mathrm{cm}^{-2}\right)$ & FF & APCE (\%) & Band gap (eV) & Conductivity $\left(\mathrm{S} \mathrm{cm}{ }^{-1}\right)$ \\
\hline BdAPbI $_{4}$ & 1.082 & 0.870 & 2.894 & 0.430 & 24.2 & 2.37 & $1.3 \times 10^{-5}$ \\
HdAPbI $_{4}$ & 0.592 & 0.725 & 1.735 & 0.471 & 8.1 & 2.44 & $1.2 \times 10^{-5}$ \\
OdAPbI $_{4}$ & 0.012 & 0.732 & 0.047 & 0.340 & 0.23 & 2.55 & $1.2 \times 10^{-5}$ \\
MAPbI $_{3}$ & 2.117 & 0.805 & 5.858 & 0.449 & 31.2 & 1.56 (ref. 19) & $1.3 \times 10^{-4}$
\end{tabular}

perovskite solar cells were made by a one step process under ambient conditions, and the highest published efficiency for this type of solar cells is just over $9 \%$ (ref. 3) under controlled glove box condition. In our previous report, an efficiency of $7.4 \%$ was obtained for $\mathbf{M A P b I}_{3}$ at much lower humidity; ${ }^{19}$ this indicates a high dependence of $\mathbf{M A P b I}_{3}$ based solar cells to humidity. The current-voltage characterization details are presented in Table 2 and Fig. 4.

Due to the differences in recombination loss and in light absorption efficiencies there is no simple correlation between the band gap of the $2 \mathrm{D}$ materials and the resulting open circuit voltage recorded from the corresponding solar cells. Incident photon-to-current conversion efficiencies (IPCE)s are presented in Fig. 6c. These curves are in good agreement with the absorption spectra and estimated band gaps. For sake of simplicity the effect of light harvesting efficiency can be omitted from the IPCE data resulting in absorbed photon-to-current conversion efficiency (APCE). APCEs for the solar cells based on BdAPbI $_{4}$ were $24.2 \%$, substantially lower than the $31.2 \%$ obtained for cells based on $\mathbf{M A P b I}_{3}$. This trend in APCE resulted in $8.1 \%$ and $0.23 \%$ for cells based on $\mathbf{H d A P b I}_{4}$ and $\mathbf{O d A P b I}_{4}$, respectively. The general conclusion is that the bulkier cation the less suitable 2D perovskite material for solar cell applications. Very poor photovoltaic performance was obtained for solar cells based on $\mathbf{O d A P b I}_{4}$, although this material shows similar electrical conductivity as and a slightly larger band gap than the other 2D materials. Most likely this can be linked to the relatively large interplane distances not being compatible with the small pore size in the mesoporous $\mathrm{TiO}_{2}$ substrate.

Relating the IPCE values to conductivity, we can estimate that conductivity between structural planes for the three related perovskites follows $\mathbf{B d A P b I} \mathbf{H}_{4}>\mathbf{H d A P b I} \mathbf{H}_{4} \gg$ OdAPbI $_{4}$. These findings suggest that charge-injection or charge-collection efficiency is a determining factor for the difference in the solar cell performance observed. The CV and UPS data indicate that both electron injection or hole injection are energetically favorable, which suggests that charge recombination may limit charge collection in solar cells that incorporate these materials.

The $I-V$-characterizations were performed at $10 \mathrm{mV} \mathrm{s}^{-1}$ to reduce hysteresis effect, ${ }^{40}$ but a small hysteresis has been observed for the 2D-based solar cells. Ion migration is suggested as one of the origins for the hysteresis in the perovskite solar cells. ${ }^{20}$ Since in these $2 \mathrm{D}$ perovskite the cations are welldefined by X-ray single crystallography, it can be suggested that this portion of hysteresis is much lower in these 2D based perovskite solar cells compare to $\mathbf{M A P b I}_{3}$ solar cells (see Fig. S8 and Table S11†).
$I-V$ measurements of the solar cells were repeated after 48 and 96 hours (see Table S9†). For BdAPbI ${ }_{4}$ and $\mathbf{H d A P b I}{ }_{4}$, the solar cells efficiency drops from $1.082 \%$ to $0.798 \%$ and from $0.592 \%$ to $0.339 \%$ after 4 days respectively, while for $\mathbf{M A P b I}_{3}$ the efficiency was reduced from $2.11 \%$ to $0.03 \%$ over that same period. This is further evidence for the increased stability of the 2D perovskite solar cells in comparison to $\mathbf{M A P b I} \mathbf{I}_{3}$ solar cells under ambient conditions.

Previous attempts at producing low dimensional lead(II) halide solar cells resulted in materials with low conductivity and a non-perovskite structure. ${ }^{19}$ Here, we attribute the increased efficiency of solar cells with diammonium cations to the decreased distance between lead(II) halide planes and the rigid dication. For comparison, the molecular volume of two ethylammonium cations $\left(\mathrm{CH}_{3} \mathrm{CH}_{2} \mathrm{NH}_{3}\right)^{+}$is similar to that of one butyldiammonium cation, $\left[\mathrm{NH}_{3}\left(\mathrm{CH}_{2}\right)_{4} \mathrm{NH}_{3}\right]^{2+}$; however, the dication has fewer rotational degrees of freedom than would be obtained for two monocations. The dications form more crystalline materials with higher conductivities, because they have fewer degrees of freedom during crystallization and in the fully formed materials.

Methylammonium lead iodide has been used as top layer in tandem cells. ${ }^{41,42}$ Although representing a novel idea, methylammonium lead iodide with a $1.5 \mathrm{eV}$ band gap is not an optimal choice for harvesting high energy photons. For instance for an ideal tandem cell incorporating silicon with a band gap of $1.1 \mathrm{eV}$, a top layer of a perovskite with a $1.8 \mathrm{eV}$ band gap is required to render a $42 \%$ theoretical efficiency. ${ }^{43}$ The present $2 \mathrm{D}$ perovskite materials with $2.3-2.5 \mathrm{eV}$ band gaps are highly appropriate to combine with CdTe $(1.5 \mathrm{eV})$ to reach a $35 \%$ theoretical efficiency. ${ }^{43}$ Energy diagrams show a better coupling capability of the 2D materials for tandem devices than $\mathbf{M A P b I}_{3}$. There is sufficient driving force between the solar cell components suggesting no problems in association with charge injection for such solar cells. In addition the higher energies of the conduction bands in these materials in combination with their higher stability may make them suitable candidates for reductive photoelectrochemical studies or photocatalysis.

\section{Conclusions}

We have synthesized and characterized three new 2D alkyl diammonium lead iodide materials. Detailed structural information of these materials has been reported based on single crystal X-ray diffraction. Diammonium cations incorporated in to the structures of these materials serve as a bridge between the inorganic lead iodide planes. Powder diffraction patterns of the 
bulk materials match single crystal data quite well, which indicates that the single crystal structures of the materials are representative for the bulk. UV-visible absorption spectra of the materials render optical properties and band gaps of $2.37 \mathrm{eV}$, $2.44 \mathrm{eV}$ and $2.55 \mathrm{eV}$ for $\mathbf{B d A P b I}_{4}, \mathbf{H d A P b I}_{\mathbf{4}}$ and $\mathbf{O d A P b I}$, respectively. Energy band diagrams of the materials were obtained from cyclic voltammetry and from ultraviolet photoelectron spectroscopy giving complementary information. Thermal stability was recorded for $\mathbf{B d A P b I} \mathbf{A}_{\mathbf{4}}$ and $\mathbf{H d A P} \mathbf{A b} \mathbf{I}_{\mathbf{4}}$ in excess of $200{ }^{\circ} \mathrm{C}$. Solar cells based on the $2 \mathrm{D}$ perovskite material BdAPbI $_{4}$ had a power conversion efficiency of $1.082 \%$, as compared to $2.1 \%$ for $\mathbf{M A P b I}_{3}$ based solar cells manufactured under the same (humid) conditions. The current study has offered insights into the effects of structural dimensionality on physical properties as well as their applicability in solar cells. In further work, we will focus on optimization of the structural networks in lead-free materials for photovoltaic applications.

\section{Acknowledgements}

This work was supported by Swedish Government through "STandUP for ENERGY", The Swedish Energy Agency, The Swedish Research Council, Knut \& Alice Wallenberg Foundation, and the Korea-Sweden Collaborative Research Program through the National Research Foundation of Korea (NRF) funded by the Ministry of Science, ICT and Future Planning (NRF-2014K1A3A1A47067328).

\section{References}

1 A. Kojima, K. Teshima, Y. Shirai and T. Miyasaka, J. Am. Chem. Soc., 2009, 131, 6050-6051.

2 J.-H. Im, C.-R. Lee, J.-W. Lee, S.-W. Park and N.-G. Park, Nanoscale, 2011, 3, 4088-4093.

3 H.-S. Kim, C.-R. Lee, J.-H. Im, K.-B. Lee, T. Moehl, A. Marchioro, S.-J. Moon, R. Humphry-Baker, J.-H. Yum, J. E. Moser, M. Gratzel and N.-G. Park, Sci. Rep., 2012, 2, 591. 4 M. M. Lee, J. Teuscher, T. Miyasaka, T. N. Murakami and H. J. Snaith, Science, 2012, 338, 643-647.

5 J. Burschka, N. Pellet, S.-J. Moon, R. Humphry-Baker, P. Gao, M. K. Nazeeruddin and M. Gratzel, Nature, 2013, 499, 316319.

6 M. Liu, M. B. Johnston and H. J. Snaith, Nature, 2013, 501, 395-398.

7 W. S. Yang, J. H. Noh, N. J. Jeon, Y. C. Kim, S. Ryu, J. Seo and S. I. Seok, Science, 2015, 348, 1234-1237.

8 D. Bi, W. Tress, M. I. Dar, P. Gao, J. Luo, C. Renevier, K. Schenk, A. Abate, F. Giordano, J.-P. Correa Baena, J.-D. Decoppet, S. M. Zakeeruddin, M. K. Nazeeruddin, M. Grätzel and A. Hagfeldt, Sci. Adv., 2016, 2(1), e1501170.

9 http://www.nrel.gov/ncpv/images/efficiency_chart.jpg.

10 N. J. Jeon, J. H. Noh, Y. C. Kim, W. S. Yang, S. Ryu and S. I. Seok, Nat. Mater., 2014, 13, 897-903.

11 L. N. Quan, M. Yuan, R. Comin, O. Voznyy, E. M. Beauregard, S. Hoogland, A. Buin, A. R. Kirmani, K. Zhao, A. Amassian, D. H. Kim and E. H. Sargent, J. Am. Chem. Soc., 2016, 138, 2649-2655.
12 A. Poglitsch and D. Weber, J. Chem. Phys., 1987, 87, 63736378.

13 F. Somma, K. Nitsch, C. Giampaolo, A. R. Phani and S. Santucci, Superficies Vacio, 1999, 9, 3.

14 I. Chung, J.-H. Song, J. Im, J. Androulakis, C. D. Malliakas, H. Li, A. J. Freeman, J. T. Kenney and M. G. Kanatzidis, J. Am. Chem. Soc., 2012, 134, 8579-8587.

15 C. C. Stoumpos, C. D. Malliakas and M. G. Kanatzidis, Inorg. Chem., 2013, 52, 9019-9038.

16 C. C. Stoumpos, L. Frazer, D. J. Clark, Y. S. Kim, S. H. Rhim, A. J. Freeman, J. B. Ketterson, J. I. Jang and M. G. Kanatzidis, J. Am. Chem. Soc., 2015, 137, 6804-6819.

17 D. B. Mitzi, C. A. Feild, Z. Schlesinger and R. B. Laibowitz, J. Solid State Chem., 1995, 114, 159-163.

18 T. Baikie, Y. Fang, J. M. Kadro, M. Schreyer, F. Wei, S. G. Mhaisalkar, M. Graetzel and T. J. White, J. Mater. Chem. A, 2013, 1, 5628-5641.

19 M. Safdari, A. Fischer, B. Xu, L. Kloo and J. M. Gardner, J. Mater. Chem. A, 2015, 3, 9201-9207.

20 J. M. Azpiroz, E. Mosconi, J. Bisquert and F. De Angelis, Energy Environ. Sci., 2015, 8, 2118-2127.

21 C. Eames, J. M. Frost, P. R. F. Barnes, B. C. O'Regan, A. Walsh and M. S. Islam, Nat. Commun., 2015, 6, 7497.

22 V. D'Innocenzo, G. Grancini, M. J. P. Alcocer, A. R. S. Kandada, S. D. Stranks, M. M. Lee, G. Lanzani, H. J. Snaith and A. Petrozza, Nat. Commun., 2014, 5, 3586.

23 K. Galkowski, A. Mitioglu, A. Miyata, P. Plochocka, O. Portugall, G. E. Eperon, J. T.-W. Wang, T. Stergiopoulos, S. D. Stranks, H. J. Snaith and R. J. Nicholas, Energy Environ. Sci., 2016, 9, 962-970.

24 S. Pathak, A. Sepe, A. Sadhanala, F. Deschler, A. Haghighirad, N. Sakai, K. C. Goedel, S. D. Stranks, N. Noel, M. Price, S. Hüttner, N. A. Hawkins, R. H. Friend, U. Steiner and H. J. Snaith, ACS Nano, 2015, 9, 2311-2320.

25 J. Yang, B. D. Siempelkamp, E. Mosconi, F. De Angelis and T. L. Kelly, Chem. Mater., 2015, 27, 4229-4236.

26 R. K. Misra, S. Aharon, B. Li, D. Mogilyansky, I. Visoly-Fisher, L. Etgar and E. A. Katz, J. Phys. Chem. Lett., 2015, 6, 326-330.

27 T. M. Koh, K. Fu, Y. Fang, S. Chen, T. C. Sum, N. Mathews, S. G. Mhaisalkar, P. P. Boix and T. Baikie, J. Phys. Chem. C, 2014, 118, 16458-16462.

28 D. B. Mitzi, K. Chondroudis and C. R. Kagan, Inorg. Chem., 1999, 38, 6246-6256.

29 D. B. Mitzi, Inorg. Chem., 2000, 39, 6107-6113.

30 K. Liang, D. B. Mitzi and M. T. Prikas, Chem. Mater., 1998, 10, 403-411.

31 D. H. Cao, C. C. Stoumpos, O. K. Farha, J. T. Hupp and M. G. Kanatzidis, J. Am. Chem. Soc., 2015, 137, 7843-7850.

32 I. C. Smith, E. T. Hoke, D. Solis-Ibarra, M. D. McGehee and H. I. Karunadasa, Angew. Chem., Int. Ed., 2014, 53, 1123211235.

33 B. A. B. S. Apex II, 2013.2-0, Bruker AXS Inc., Madison, WI, USA, 2013.

34 J. Tauc, R. Grigorovici and A. Vancu, Phys. Status Solidi B, 1996, 15, 10.

35 M. De Bastiani, V. D'Innocenzo, S. D. Stranks, H. J. Snaith and A. Petrozza, APL Mater., 2014, 2, 081509. 
36 W. Zhang, M. Saliba, D. T. Moore, S. K. Pathak, M. T. Hörantner, T. Stergiopoulos, S. D. Stranks, G. E. Eperon, J. A. Alexander-Webber, A. Abate, A. Sadhanala, S. Yao, Y. Chen, R. H. Friend, L. A. Estroff, U. Wiesner and H. J. Snaith, Nat. Commun., 2015, 6, 6142.

37 T. Umebayashi, K. Asai, T. Kondo and A. Nakao, Phys. Rev. B: Condens. Matter Mater. Phys., 2003, 67, 155405.

38 R. Lindblad, D. Bi, B.-w. Park, J. Oscarsson, M. Gorgoi, H. Siegbahn, M. Odelius, E. M. J. Johansson and H. Rensmo, J. Phys. Chem. Lett., 2014, 5, 648-653.

39 E. A. Muljarov, S. G. Tikhodeev, N. A. Gippius and T. Ishihara, Phys. Rev. B: Condens. Matter Mater. Phys., 1995, 51, 14370-14378.
40 E. L. Unger, E. T. Hoke, C. D. Bailie, W. H. Nguyen, A. R. Bowring, T. Heumuller, M. G. Christoforo and M. D. McGehee, Energy Environ. Sci., 2014, 7, 3690-3698.

41 C. D. Bailie, M. G. Christoforo, J. P. Mailoa, A. R. Bowring, E. L. Unger, W. H. Nguyen, J. Burschka, N. Pellet, J. Z. Lee, M. Gratzel, R. Noufi, T. Buonassisi, A. Salleo and M. D. McGehee, Energy Environ. Sci., 2015, 8, 956-963.

42 P. Loper, S.-J. Moon, S. Martin de Nicolas, B. Niesen, M. Ledinsky, S. Nicolay, J. Bailat, J.-H. Yum, S. De Wolf and C. Ballif, Phys. Chem. Chem. Phys., 2015, 17, 1619-1629. 43 A. D. Vos, J. Phys. D: Appl. Phys., 1980, 13, 839. 